\title{
Early optical and millimeter observations of GRB 030226 afterglow
}

\author{
S. B. Pandey ${ }^{1}$, R. Sagar ${ }^{1,2}$, G. C. Anupama ${ }^{2}$, D. Bhattacharya ${ }^{3}$, D. K. Sahu ${ }^{2,4}$, \\ A. J. Castro-Tirado ${ }^{5}$, and M. Bremer ${ }^{6}$
}

\author{
1 State Observatory, Manora Peak, Nainital - 263 129, Uttaranchal, India \\ 2 Indian Institute of Astrophysics, Bangalore - 560 034, India \\ 3 Raman Research Institute, Bangalore - 560 080, India \\ ${ }^{4}$ Center for Research \& Education in Science \& Technology, Hosakote, Bangalore - 562 114, India \\ ${ }^{5}$ Instituto de Astrofísica de Andalucía, PO Box 03004, 18080 Granada, Spain \\ ${ }^{6}$ IRAM, 300 rue de la Piscine, Dom. Universitaire, 38406 Saint Martin d'Hères, France
}

Received 15 October 2003 / Accepted 7 January 2004

\begin{abstract}
The CCD magnitudes in Johnson $U B V$ and Cousins $R I$ photometric passbands for the afterglow of the long duration GRB 030226 are presented. Upper limits of a few mJy to millimeter wave emission at the location of optical are obtained over the first two weeks. The optical data presented here, in combination with other published data on this afterglow, show an early $R$ band flux decay slope of $0.77 \pm 0.04$, steepening to $2.05 \pm 0.04$ about $0.65 \pm 0.03$ day after the burst. Interpreted as the "jet break", this indicates a half opening angle of $\sim 3.2$ degree for the initial ejection, for an assumed ambient density of $\sim 1 \mathrm{~cm}^{-3}$. Broadband spectra show no appreciable evolution during the observations, and indicate the presence of synchrotron cooling frequency $v_{\mathrm{c}}$ near the upper edge of the optical band. From the broadband spectra we derive an electron energy distribution index $p=2.07 \pm 0.06$ and an intrinsic extinction $E(B-V) \sim 0.17$. Millimeter upper limits are consistent with these derived parameters.
\end{abstract}

Key words. gamma rays: bursts - gamma rays: observations

\section{Introduction}

Multi-wavelength observations of an afterglow of a Gamma Ray Burst (GRB), an event which emits $\sim 10^{51}-10^{53}$ ergs of energy in a few seconds, provide valuable information about its progenitor (Mészáros 2002). Optical observations occupy the central place in such studies as they provide information about distance as well as isotropic/nonisotropic nature of the emission (e.g. Sagar 2002). In this paper we present the early optical and millimeter-wave observations of the long duration (>100 s) GRB 030226 ( $\equiv \mathrm{H} 10893)$. It was detected by HETE FREGATE, WXM, and soft X-ray camera (SXC) instruments at $t_{0}=03^{\mathrm{h}} 46^{\mathrm{m}} 31^{\mathrm{s}} .99$ UT on 26th Feb. 2003 (Suzuki et al. 2003). Fox et al. (2003) discovered its optical afterglow (OA) within the HETE error circle at $\alpha=11^{\mathrm{h}} 33^{\mathrm{m}} 04^{\mathrm{s}} .9, \delta=+25^{\circ} 53^{\prime} 55^{\prime \prime}$. 6 (J2000) with an uncertainty of $0 . .5$ in each coordinate (Price et al. 2003). X-ray afterglow of the GRB 030226 was discovered by Chandra X-ray observatory $\sim 37.1 \mathrm{~h}$ after the burst at the OT position (Pedersen et al. 2003). Spectroscopic observations performed by Ando et al. (2003) on 2003 Feb. 26.31 UT yield a value of redshift $z \sim 2.1$, which was later refined to 1.99. VLT spectroscopic observations indicate $z=1.986 \pm 0.001$ or larger using FeII, AlII and CVI absorption lines (Greiner et al. 2003a). Based on Keck II high resolution spectra, Price et al. (2003) calculated the redshift systems at $z=1.043,1.948$ and 1.961. Chornock \& Fillippenko (2003), using low resolution spectrograph on Keck I, deduce that the highest redshift system is associated with the GRB afterglow.

Dai \& Wu (2003) modeled the GRB 030226 afterglow light curve and concluded that a discontinuous jump in the ambient density is indicated by the data. However this work made use of the preliminary magnitude estimates communicated in the GRB Coordinates Network (GCN), which show a large scatter. This is most probably due to the use of inhomogeneous photometric calibrations: those given by Garnavich et al. (2003) and Henden (2003) differ by 0.06 and $0.01 \mathrm{mag}$ in $R_{\mathrm{c}}$ for two important comparison stars in the field, designated $A$ and $B$ respectively. For reliable determination of parameters from the light curve of the OA, consistent and secure photometric calibrations are needed. We provide this by imaging PG0918+029 standard region of Landolt (1992) along with GRB 030226 field. 
Table 1. Millimeter wave observations of the GRB 030226 afterglow.

\begin{tabular}{cccll}
\hline \hline $\begin{array}{c}\text { Start } \\
\text { time }\end{array}$ & $\begin{array}{c}\text { End } \\
\text { time }\end{array}$ & $\begin{array}{c}\text { Center } \\
\text { time }\end{array}$ & $\begin{array}{l}\text { Frequency } \\
(\mathrm{GHz})\end{array}$ & $\begin{array}{l}\text { Flux } \\
\text { center }(\mathrm{mJy})\end{array}$ \\
\hline Feb. 27.866 & 28.045 & 27.956 & 85.283 & $+0.54 \pm 0.31$ \\
Feb. 27.866 & 28.045 & 27.956 & 220.311 & $-0.90 \pm 1.96$ \\
Mar. 02.107 & 02.291 & 02.199 & 89.199 & $-0.50 \pm 0.76$ \\
Mar. 08.178 & 08.233 & 08.206 & 91.995 & $-1.01 \pm 0.70$ \\
Mar. 08.178 & 08.233 & 08.206 & 232.032 & $-0.83 \pm 3.45$ \\
Mar. 10.166 & 10.197 & 10.182 & 90.782 & $-0.43 \pm 0.84$ \\
Mar. 10.166 & 10.197 & 10.182 & 221.903 & $-4.00 \pm 3.86$ \\
Mar. 11.908 & 12.014 & 11.961 & 114.676 & $+0.73 \pm 0.68$ \\
Mar. 11.908 & 12.014 & 11.961 & 230.973 & $-2.13 \pm 1.51$ \\
Mar. 14.942 & 15.126 & 15.034 & 92.097 & $+0.23 \pm 0.34$ \\
Mar. 14.942 & 15.126 & 15.034 & 219.006 & $-0.25 \pm 1.12$ \\
\hline
\end{tabular}

\section{Observations and data reductions}

The millimeter wave and broad band optical observations of the GRB 030226 afterglow are described in the following sections.

\subsection{Millimeter wave observations}

The GRB 030226 afterglow was observed with the Plateau de Bure Interferometer (Guilloteau et al. 1992) in a sixantenna extended configuration on the dates listed in Table 1. Flux calibration includes a correction for atmospheric decorrelation which has been determined with a UV plane point source fit of the phase calibration quasar $1156+295$. The carbon star MWC349 has been used as a primary flux calibrator due to its well-known millimeter spectral properties (see, e.g., Schwarz 1980). There is no detection of millimeter wave emission at the location of OA during two weeks of observations after the burst (see Table 1).

\subsection{Optical observations and calibrations}

The broad band Johnson $U B V$ and Cousins $R I$ observations of the OA were carried out from 26 to 27 Feb. 2003 using 2-m Himalayan Chandra Telescope (HCT) of the Indian Astronomical Observatory (IAO), Hanle and the 104-cm Sampurnanand telescope of the State Observatory, Nainital. At Nainital, the CCD chip of size $2048 \times 2048$ pixel $^{2}$ covers a field of $\sim 13^{\prime} \times 13^{\prime}$ with one pixel corresponding to 0 '. $^{\prime} 38$ square on the sky. The gain and read out noise of the CCD camera are $10 \mathrm{e}^{-} / \mathrm{ADU}$ and $5.3 \mathrm{e}^{-}$respectively. At Hanle, the CCD used was $1024 \times 1024$ pixel $^{2}$, the entire chip covering a field of $\sim 4$ ". $7 \times 4$.' 7 on the sky. It has a read out noise of $11 \mathrm{e}^{-}$ and gain is $4.8 \mathrm{e}^{-} /$ADU. During good photometric sky conditions, the CCD $B V R I$ observations of the OA field along with Landolt (1992) standard PG0918+029 region were obtained on 26/27 Feb. 2003 for calibration purposes. Several twilight flat field and bias frames were also obtained during the observing run for the CCD calibrations.

ESO MIDAS, NOAO IRAF and DAOPHOT-II softwares were used for cleaning and image processing of CCD frames. At Nainital, on the night of 26/27 Feb. 2003, atmospheric
Table 2. The identification number (ID), $(\alpha, \delta)$ for epoch 2000, standard $V,(B-V),(V-R)$ and $(V-I)$ photometric magnitudes of the stars in the GRB 030226 region are given. Garnavich von Braun \& Stanek (2003) stars $A$ and $B$ are the stars 6 and 4 respectively. Number of observations taken in $B, V, R$ and $I$ filters are 3, 4, 4 and 3 respectively.

\begin{tabular}{ccccccc}
\hline \hline ID & $\begin{array}{c}\alpha_{2000} \\
\text { (h m s) }\end{array}$ & $\begin{array}{c}\delta_{2000} \\
\text { (deg m s })\end{array}$ & $\begin{array}{c}V \\
(\mathrm{mag})\end{array}$ & $\begin{array}{c}B-V \\
(\mathrm{mag})\end{array}$ & $\begin{array}{c}V-R \\
(\mathrm{mag})\end{array}$ & $\begin{array}{c}V-I \\
(\mathrm{mag})\end{array}$ \\
\hline 1 & 113244 & 255852 & 17.01 & 0.81 & 0.51 & 0.96 \\
2 & 113246 & 255656 & 16.72 & 1.12 & 0.64 & 1.20 \\
3 & 113247 & 255158 & 16.10 & 0.62 & 0.38 & 0.75 \\
4 & 113259 & 255238 & 17.45 & 0.56 & 0.35 & 0.68 \\
5 & 113300 & 255713 & 15.32 & 0.60 & 0.36 & 0.71 \\
6 & 113303 & 255130 & 16.14 & 1.16 & 0.68 & 1.24 \\
7 & 113305 & 255812 & 17.29 & 0.80 & 0.45 & 0.88 \\
8 & 113318 & 255030 & 15.87 & 0.81 & 0.48 & 0.91 \\
9 & 113319 & 254837 & 16.43 & 0.66 & 0.41 & 0.79 \\
10 & 113328 & 255107 & 16.46 & 1.07 & 0.65 & 1.22 \\
\hline
\end{tabular}

extinction coefficients determined from the observations of PG0918+029 bright stars are 0.57, 0.32, 0.20, 0.17 and $0.15 \mathrm{mag}$ in $U, B, V, R$ and $I$ filters respectively. The five standard stars in the PG0918+029 region cover a range of $-0.29<$ $(V-I)<1.11$ in color and of $12.3<V<14.5$ in brightness. They yield the following transformation equations:

$$
\begin{aligned}
& u_{\mathrm{CCD}}=U-(0.084 \pm 0.01)(U-B)+(6.83 \pm 0.01) \\
& b_{\mathrm{CCD}}=B-(0.062 \pm 0.01)(B-V)+(4.79 \pm 0.01) \\
& v_{\mathrm{CCD}}=V-(0.034 \pm 0.01)(B-V)+(4.34 \pm 0.01) \\
& r_{\mathrm{CCD}}=R-(0.034 \pm 0.01)(V-R)+(4.24 \pm 0.01) \\
& i_{\mathrm{CCD}}=I-(0.048 \pm 0.01)(V-R)+(4.74 \pm 0.01)
\end{aligned}
$$

where $U, B, V, R, I$ are standard magnitudes and $u_{\mathrm{CCD}}, v_{\mathrm{CCD}}, b_{\mathrm{CCD}}, r_{\mathrm{CCD}}$ and $i_{\mathrm{CCD}}$ represent the instrumental magnitudes normalized for $1 \mathrm{~s}$ of exposure time and corrected for atmospheric extinction. The colour coefficients, zero-points and errors in them are determined by fitting least square linear regressions to the data points. Using these transformations, $B V R I$ photometric magnitudes of 10 secondary standard stars are determined in the GRB 030226 field and their average values are listed in Table 2 . The $(X, Y)$ CCD pixel coordinates are converted into $\alpha_{2000}, \delta_{2000}$ values using the astrometric positions given by Henden (2003). All these stars, observed 3 to 4 times in a filter, have internal photometric accuracy better than 0.01 mag. A comparison between our photometry and that of Henden (2003) yields zero-point differences of $0.024 \pm 0.035,0.013 \pm 0.022,0.018 \pm 0.021$ and $-0.021 \pm 0.036 \mathrm{mag}$ in $B, V, R$ and $I$ filters respectively. These numbers are based on the comparison of secondary stars having range in brightness from $V=15$ to $17.5 \mathrm{mag}$. There is no colour dependence in the photometric differences. We therefore conclude that photometric calibration used in this work is secure.

Our observations started about 0.5 day after the burst and are valuable for dense temporal coverage of the light curve. Several short exposures up to a maximum of $30 \mathrm{~min}$ were generally given while imaging the OA (see Table 3). In order to improve the signal-to-noise ratio of the OA, the data 
Table 3. CCD $U B V R I$ broad band optical photometric observations of the GRB 030226 afterglow. At Hanle, 2-m HCT was used while at Nainital, 104-cm Sampurnanand optical telescope was used.

\begin{tabular}{|c|c|c|c|}
\hline $\begin{array}{l}\text { Date (UT) of } \\
2003 \text { February }\end{array}$ & $\begin{array}{l}\text { Magnitude } \\
\text { (mag) }\end{array}$ & $\begin{array}{l}\text { Exposure time } \\
\text { (s) }\end{array}$ & Telescope \\
\hline & $U-$ passband & & \\
\hline 26.7729 & $\begin{array}{c}20.17 \pm 0.11 \\
B-\text { passband }\end{array}$ & 1800 & $104-\mathrm{cm}$ \\
\hline 26.6969 & $20.42 \pm 0.08$ & $2 \times 300$ & $104-\mathrm{cm}$ \\
\hline 26.7326 & $20.31 \pm 0.07$ & 600 & $104-\mathrm{cm}$ \\
\hline 26.8479 & $20.82 \pm 0.08$ & 600 & $104-\mathrm{cm}$ \\
\hline 26.6837 & $\begin{array}{c}V-\text { passband } \\
19.87 \pm 0.04\end{array}$ & $2 \times 300$ & $104-\mathrm{cm}$ \\
\hline 26.7335 & $20.17 \pm 0.03$ & 900 & HCT 2-m \\
\hline 26.7410 & $20.25 \pm 0.05$ & 600 & $104-\mathrm{cm}$ \\
\hline 26.8049 & $20.31 \pm 0.05$ & 600 & $104-\mathrm{cm}$ \\
\hline 26.8172 & $20.33 \pm 0.05$ & 900 & HCT 2-m \\
\hline 26.8597 & $20.33 \pm 0.05$ & 900 & $104-\mathrm{cm}$ \\
\hline 26.9120 & $\begin{array}{c}20.68 \pm 0.11 \\
R-\text { passband }\end{array}$ & 900 & HCT 2-m \\
\hline 26.6398 & $19.60 \pm 0.06$ & 450 & HCT 2-m \\
\hline 26.6484 & $19.64 \pm 0.05$ & 700 & HCT 2-m \\
\hline 26.6575 & $19.55 \pm 0.05$ & 700 & HCT 2-m \\
\hline 26.6674 & $19.52 \pm 0.04$ & $2 \times 300$ & $104-\mathrm{cm}$ \\
\hline 26.6678 & $19.62 \pm 0.05$ & 900 & НCТ 2-m \\
\hline 26.6805 & $19.63 \pm 0.04$ & 900 & HCT 2-m \\
\hline 26.6925 & $19.67 \pm 0.04$ & 900 & НCT 2-m \\
\hline 26.7065 & $19.76 \pm 0.04$ & 900 & HCT 2-m \\
\hline 26.7216 & $19.78 \pm 0.04$ & 900 & HCT 2-m \\
\hline 26.7493 & $19.74 \pm 0.04$ & 600 & $104-\mathrm{cm}$ \\
\hline 26.8139 & $19.84 \pm 0.04$ & 600 & $104-\mathrm{cm}$ \\
\hline 26.8715 & $20.22 \pm 0.06$ & 900 & HCT 2-m \\
\hline 26.8715 & $20.11 \pm 0.04$ & 900 & $104-\mathrm{cm}$ \\
\hline 26.8968 & $20.23 \pm 0.06$ & 900 & НCT 2-m \\
\hline 26.9932 & $20.45 \pm 0.06$ & $2 \times 900$ & HCT 2-m \\
\hline 27.8074 & $21.98 \pm 0.14$ & 900 & $104-\mathrm{cm}$ \\
\hline 27.8750 & $\begin{array}{c}22.00 \pm 0.15 \\
I-\text { passband }\end{array}$ & 1800 & $104-\mathrm{cm}$ \\
\hline 26.6774 & $19.00 \pm 0.07$ & $2 \times 300$ & $104-\mathrm{cm}$ \\
\hline 26.7576 & $19.34 \pm 0.09$ & 600 & $104-\mathrm{cm}$ \\
\hline 26.7764 & $19.59 \pm 0.04$ & 900 & HCT 2-m \\
\hline 26.8229 & $19.52 \pm 0.09$ & 600 & $104-\mathrm{cm}$ \\
\hline 26.8333 & $19.51 \pm 0.06$ & 600 & HCT 2-m \\
\hline 27.8306 & $21.78 \pm 0.34$ & $2 \times 900$ & $104-\mathrm{cm}$ \\
\hline
\end{tabular}

have been binned in $2 \times 2$ pixel $^{2}$ and also several bias corrected and flat-fielded CCD images of OA field taken on a night are co-added in the same filter, when found necessary. From these images, profile-fitting magnitudes are determined using DAOPHOT-II software. To determine the difference between aperture and profile fitting magnitudes, we constructed an aperture growth curve of the well-isolated stars and used them to determine aperture (about 5 arcsec) magnitudes of the OA. They are calibrated differentially with respect to the secondary standards listed in Table 2 and the values derived in this way are given in Table 3 . The distribution of these 34 data points is $N(U, B, V, R, I)=(1,3,7,17,6)$.

The secondary standards are also used to calibrate other photometric measurements of OA published in GCN circulars by Ando et al. (2003), Covino et al. (2003),

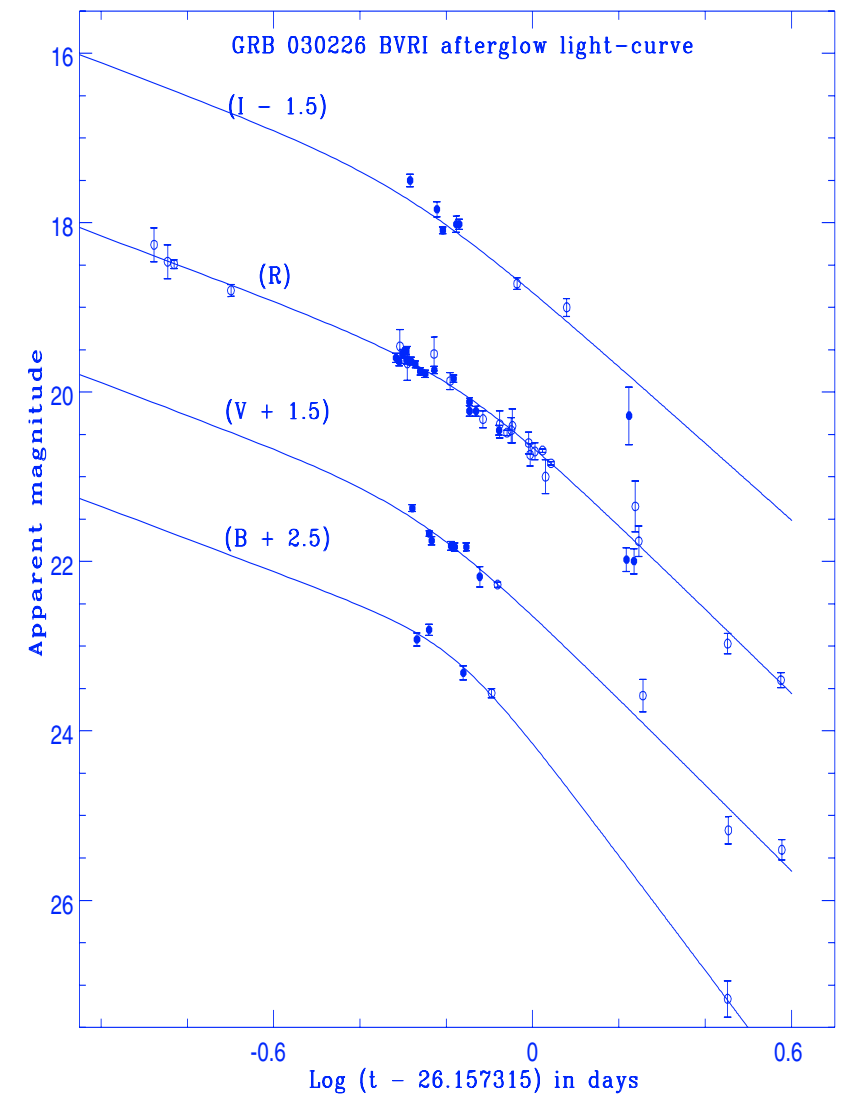

Fig. 1. Optical light curves of GRB 030226 afterglow in BVRI passbands. Marked vertical offsets are applied to avoid overlapping of data points of different passbands. The present measurements are marked by solid circles while those taken from the literature are shown by open circles. The solid curves are the least square best fitted relations for the parameters listed in Table 4.

Fatkhullin et al. (2003), Garnavich von Braun \& Stanek (2003), Greiner et al. (2003b), Guarnieri et al. (2003), Maiorano et al. (2003), Nysewander et al. (2003), Price \& Warren (2003), Rumyantsev et al. (2003a,b), Semkov (2003) and Von Braun et al. (2003). The distribution of these 27 photometric data points is $N(B, V, R, I)=(2,4,19,2)$.

\section{Optical photometric light curves}

Figure 1 shows the light curve of GRB 030226 afterglow in $B, V, R$ and $I$ passbands. Present observations in combinations with above mentioned published ones are used in the plot. The $X$-axis is $\log \Delta t\left(=t-t_{0}\right)$. Here $t$ is the time of observation and $t_{0}=2003$ Feb. 26.157315 UT is the burst epoch.

The light curve appears to steepen after $\Delta t>0.6$ day in $R$ passband. Lack of early observations makes it difficult to see this transition in $B V I$ light curves. To determine the flux decay constants and the break time, we fitted the following empirical function (see Rhoads \& Fruchter 2001) which represents a broken power-law, to the $R$ band light curve.

$m=m_{b}+\frac{2.5}{s}\left[\log _{10}\left\{\left(t / t_{b}\right)^{\alpha_{1} s}+\left(t / t_{b}\right)^{\alpha_{2} s}\right\}-\log _{10}(2)\right]$

where $\alpha_{1}$ and $\alpha_{2}$ are asymptotic power-law slopes at early and late times with $\alpha_{1}<\alpha_{2}$ and $s>0$ controls the sharpness of 
Table 4. Fitted parameters of GRB 030226 afterglow. The sharpness parameter $s=3$ is fixed. Large errors in derived parameters of $I$ band may be due to sparse data-set.

\begin{tabular}{ccccc}
\hline \hline $\begin{array}{c}\text { Fitted } \\
\text { parameters }\end{array}$ & $\begin{array}{c}B \\
\text { band }\end{array}$ & $\begin{array}{c}V \\
\text { band }\end{array}$ & $\begin{array}{c}R \\
\text { band }\end{array}$ & $\begin{array}{c}I \\
\text { band }\end{array}$ \\
\hline$\alpha_{1}$ & $\ldots .$. & $\ldots$. & $0.77 \pm 0.04$ & $\ldots .$. \\
$\alpha_{2}$ & $2.71 \pm 0.17$ & $2.03 \pm 0.07$ & $1.99 \pm 0.06$ & $1.82 \pm 0.34$ \\
$t_{b}$ & $0.67 \pm 0.06$ & $0.56 \pm 0.07$ & $0.69 \pm 0.04$ & $0.52 \pm 0.32$ \\
$m_{b}$ & $20.69 \pm 0.16$ & $20.08 \pm 0.19$ & $20.02 \pm 0.06$ & $19.24 \pm 0.95$ \\
$\chi^{2} /$ d.o.f & 2.59 & 3.82 & 2.06 & 5.13 \\
\hline
\end{tabular}

the break, a larger $s$ implying a sharper break. $m_{b}$ is the magnitude at the cross-over time $t_{b}$. The function describes a light curve falling as $t^{-\alpha_{1}}$ at $t<t_{b}$ and $t^{-\alpha_{2}}$ at $t>t_{b}$. The results of the fit are presented in Table 4. The value of $s$ has been fixed at 3 , which helped to achieve the minimum $\chi^{2}$. The early time $R$ band data of the OA presented by Ando et al. (2003), Price $\&$ Warren (2003) and Von Braun et al. (2003) were based on nearby USNO A-2.0 stars in the GRB 030226 field which are brighter by $0.4-0.5 \mathrm{mag}$ than that determined from our calibration. It is significant to note that once this difference in calibration is accounted for, there appears to be no sign of the rebrightening of the OA around 0.5 day after the burst, contrary to the claim by Dai \& Wu (2003). The $R$ band data can be well fitted in terms of broken power law using Eq. (1). Due to lack of early data points in $B, V$ and $I$ passbands, in carrying out fits for these bands we fixed the value of $\alpha_{1}$ at 0.77 , as determined from $R$-band. These results are also tabulated in Table 4 . The derived value of $\alpha_{2}$ in $B$ band is larger than in other bands, but this may be due to sparse late time data points; the deviation is within 3 sigma of the average value of deviation in $\alpha_{2}$. In Fig. 1 the best fit light curves obtained in this way for $B V R I$ passbands are shown. It can be seen that the observations presented here lie near the jet break time, filling important gaps in the published data.

In the light of the above, we conclude that the early time light curve decay slope $\alpha_{1}$ is $0.77 \pm 0.04$ and the weighted mean values of parameters $t_{b}$ and $\alpha_{2}$ are $0.65 \pm 0.03$ and $2.05 \pm 0.04$ respectively. The value of $\alpha_{2}$ is flatter than the observed X-ray flux decay of $3.6 \pm 1.0$ (Sako \& Fox 2003) but agrees well with $\alpha_{2} \sim 2$ determined by Klose et al. (2003) and Dai \& $\mathrm{Wu}$ (2003). The value of $s=3$ indicates that the observed break in the light curve is sharp, unlike the smooth break observed in the optical light curve of GRB 990510 (Stanek et al. 1999; Harrison et al. 1999) but similar to the sharp break observed in the optical light curves of GRB 000301c (Sagar et al. 2000; Pandey et al. 2001); GRB 000926 (Harrison et al. 2001; Sagar et al. 2001a; Pandey et al. 2001); GRB 010222 (Masetti et al. 2001; Sagar et al. 2001b; Stanek et al. 2001; Cowsik et al. 2001) and GRB 011211 (Jakobsson et al. 2003).

\section{Spectral energy distribution}

Figure 2 shows the spectrum of GRB 030226 afterglow from millimeter to X-ray region. We construct the GRB 030226 afterglow spectrum at two possible epochs to cover the longest spectral range: $\Delta t=0.62$ day, an epoch near the jet break,

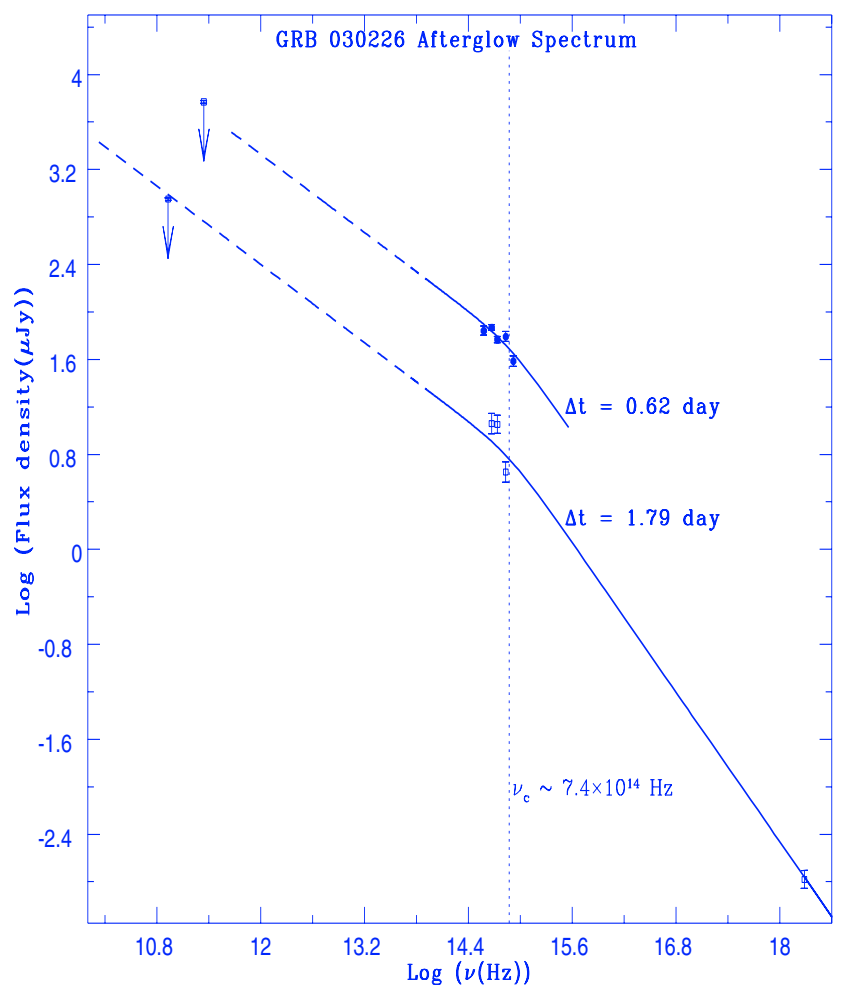

Fig. 2. Spectral energy distribution (SED) of GRB 030226 afterglow from millimeter to $\mathrm{X}$-ray passbands are shown at two marked epochs. The optical data are corrected for a small Galactic extinction of $A_{\mathrm{V}}=$ 0.06 mag and a starburst galaxy-like extinction (Calzetti et al. 1997) in the host galaxy, of amount $E(B-V)=0.17 \mathrm{mag}$. Arrows show the millimeter upper limits $(85 \mathrm{GHz}$ and $220 \mathrm{GHz})$ at $\Delta t=1.79$ day. Filled and open circles show the data at $\Delta t=0.62$ and 1.79 day respectively. The location of the cooling frequency $v_{\mathrm{c}}$ is shown by a dotted line.

and $\Delta t=1.79$ day, corresponding to the epoch of X-ray observations. We also estimate the millimeter upper limits from the present measurements for the epoch $\Delta t=1.79$ day. The reddening map provided by Schlegel et al. (1998) indicates a small value of $E(B-V)=0.02$ mag for the Galactic interstellar extinction towards the burst. We used the standard Galactic extinction reddening curve given by Mathis (1990) to convert apparent magnitudes into fluxes, with the effective wavelengths and normalizations from Fukugita et al. (1995) for UBVRI. $\mathrm{X}$-ray measurements were obtained by Pedersen et al. (2003) in $2-10 \mathrm{KeV}$ energy range. Considering mid UT as the epoch and $20 \%$ uncertainty in the measurements, we calculate the X-ray flux at $\Delta t=1.79$ day. For the epochs under study it is observed that the flux increases as the frequency decreases.

In the simple synchrotron model of the GRB afterglow, the light curves and spectral energy distributions are generally described as a power law: $F(t, v) \propto t^{-\alpha} v^{-\beta}$. The spectral slope $\beta$ depends on the location of the spectral breaks, so does the temporal slope $\alpha$, which also has an additional dependence on the dynamics of the fireball. However within each regime $\alpha$ and $\beta$ are functions only of $p$, the power law exponent of the electron Lorentz factor. For an initially collimated ejection, at late 
times when the evolution comes to be dominated by the lateral spreading of the jet, the value of $\alpha$ is expected to approach $p$. The value of $\beta$ is expected to be $p / 2$ above the cooling frequency $v_{\mathrm{c}}$ and $(p-1) / 2$ below it, down to the peak of the spectrum, in the slow cooling case (Sari et al. 1999). In the present case the post jet-break flux decay constant is determined to be $\alpha_{2}=2.05 \pm 0.04$, indicating that the electron energy distribution index $p$ must be close to this value (Rhoads 1999). At $\Delta t=0.62$ day, the fitted spectrum through the optical band has spectral slope $\beta \sim 1.0$ and at $\Delta t=1.79$ day, $\beta \sim 1.2$. The determined values of $\beta$ at both epochs are similar and are consistent with the measured $\alpha_{2}=2.05 \pm 0.04$ if $v>v_{\mathrm{c}}$ in the optical band. However this predicts a pre jet-break decay constant $\alpha_{1}$ around 1.1, much steeper than the observed value of $0.77 \pm 0.04$. So the cooling frequency is unlikely to be below the optical band. A consistent picture, including the measured $\mathrm{X}$-ray flux, can be obtained if the cooling frequency is located just above the optical band, and the intrinsic optical spectrum of the afterglow is flatter, with a slope $\sim 0.55$. Extinction along the line of sight would steepen the optical spectrum, and could explain the observed $\beta \sim 1$. The estimated Galactic extinction of $A_{\mathrm{V}}=0.06$ mag does not change the slope much, so we attribute the rest of the extinction to the host galaxy of the GRB. Assuming $p=2.1$, we find that $E(B-V) \approx 0.15 \mathrm{mag}$ in the host galaxy, with an extinction law similar to that in starburst galaxies (Calzetti et al. 1997), can reproduce the expected spectrum. Assuming that the cooling frequency $v_{\mathrm{c}}$ is located $\sim 7 \times 10^{14} \mathrm{~Hz}$, the result is consistent also with the $\mathrm{X}$-ray flux observed at 1.79 day (see Fig. 2). The cooling frequency is expected to be nearly the same even in the 0.62 day spectrum since both epochs fall after the jet break. We found that a host extinction law similar to that for our galaxy (Cardelli et al. 1989) is unable to provide a consistent fit: the presence of the $2200 \AA$ bump introduces additional scatter in the data. A combined fit to spectra at both epochs, with $p$ and $v_{\mathrm{c}}$ fixed to the above values, yields the following estimate for the extinction in the host galaxy: $E(B-V) \sim 0.17 \mathrm{mag}$.

The two millimeter upper limits at $\Delta t=1.79$ day lie close to or above the extrapolation of the optical spectrum, and are therefore consistent with the above interpretation. It may be noted that the above derived parameters have an accuracy of $\sim 10-20 \%$ only, as they are based on a few available observed data points.

\section{Discussion and conclusions}

We have presented optical photometry of the afterglow of GRB 030226 in UBVRI bands from 0.5 to 2 days and millimeter observations for up to 20 days after the burst. We do not detect millimeter wave emission down to a few mJy. The overall flux decay observed in $R$ band is well understood in terms of a jet model. The flux decay constants at early and late times are $0.77 \pm 0.04$ and $2.05 \pm 0.04$ respectively, and the jet break time is $0.65 \pm 0.05$ day. These decay indices indicate a value of the electron energy distribution index $p=2.07 \pm 0.06$. Comparison of the observed broadband spectrum with that predicted by synchrotron emission model suggests an extinction $E(B-V) \sim 0.17$ mag in the host galaxy, and an extinction law similar to that found in starburst galaxies (Calzetti et al. 1997). The cooling break is inferred to be located at the upper edge of the optical band, $v_{\mathrm{c}} \sim 7 \times 10^{14} \mathrm{~Hz}$.

GRB 030226 belongs to a small subset of GRBs which display a steepening in the optical afterglow light curve (jet break) within a day after the burst. Other known cases include GRB 980519 ( 0.6 day, Jaunsen et al. 2001), GRB 010222 ( $\sim .7$ day, Stanek et al. 2001; Sagar et al. 2001b), GRB 020813 ( $\sim 0.2$ day, Bloom et al. 2002) and GRB 030329 ( $\sim 0.6$ day, Burenin et al. 2003). Of these, GRB 980519 showed a cooling break between optical and X-ray bands (Jaunsen et al. 2001), GRB 010222 had its cooling break below the optical band (Sagar et al. 2001b) and in the present case we infer the cooling break to be at the upper edge of the optical band. GRB 010222 also exhibited a hard electron energy spectrum $p<2$ (Bhattacharya 2001; Sagar et al. 2001b).

The observed fluence of $5.7 \mu \mathrm{erg} / \mathrm{cm}^{2}$ in the energy band $30-400 \mathrm{KeV}$ with the measured redshift $z=1.986 \pm 0.001$ indicates an isotropic equivalent energy release $E_{\text {iso }} \sim 6.5 \times 10^{52} \mathrm{erg}$ for $H_{0}=65 \mathrm{~km} \mathrm{~s}^{-1} \mathrm{Mpc}^{-1}$ in a $\Omega_{0}=0.3$ and $\Lambda_{0}=0.7$ cosmological model. The observed jet-break time of 0.6 day leads to an estimated jet half-opening angle of $\sim 3.2$ degree, for an assumed particle density $n=1 \mathrm{~cm}^{-3}$. The total energy output in the jet then works out to be $\sim 0.8 \times 10^{51} \mathrm{erg}$ for a $\gamma$-ray efficiency $\eta_{\gamma}=0.2$, after applying the cosmological K-correction (Bloom et al. 2001). This is close to the estimated mean energy output in Gamma Ray Bursts (Frail et al. 2001; Kulkarni et al. 2003; Berger et al. 2003), and supports the case for GRBs as standard energy reservoirs.

Acknowledgements. This research has made use of data obtained through the High Energy Astrophysics Science Archive Research Center Online Service, provided by the NASA/Goddard Space Flight Center. Thanks to anonymous referee for the useful comments.

\section{References}

Ando, M., Ohta, K., Watanabe, C., et al. 2003, GCNC, 1882, 1884 Berger, E., Kulkarni, S. R., Pooley, G., et al. 2003, Nature, 426, 154 Bhattacharya, D. 2001, BASI, 29, 107

Frail, D. A., Kulkarni, S. R., Sari R., et al. 2001, ApJ, 562, L55

Bloom, J. S., Frail, D. A., \& Sari, R. 2001, AJ, 121, 2879

Bloom, J. S., Fox, D. W., \& Hunt, M. P. 2002, GCNC, 1476

Burenin, R. A., Sunyaev, R. A., Pavlinsky, M. N., et al. 2003, Astr. Lett., 29,9

Calzetti, D. 1997, AJ, 113, 162

Cardelli, J. A., Clayton, G. C., \& Mathis, J. S. 1989, ApJ, 345, 245

Cowsik, R., Prabhu, T. P., Anupama, G. C., et al. 2001, BASI, 29, 157

Chornock, R., \& Fillippenko, A. V. 2003, GCNC 1897

Covino, S., Ghisellini, G., Malesani, D., et al. 2003, GCNC, 1909

Dai, Z. G., \& Wu, X. F. 2003, ApJ, 591, L21

Fatkhullin, T., Komarova, V., Sokolov, V., Cherepashchuk, A., \& Postnov, K. 2003, GCNC, 1925

Fox, D. W., Chen, H. W., \& Price, P. A. 2003, GCNC, 1879

Fukugita, M., Shimasaku, K., \& Ichikawa, T. 1995, PASP, 107, 945

Garnavich, P., Von Braun, K., \& Stanek, K. 2003, GCNC 1885

Greiner, J., Guenther, E., Klose., S., \& Schwarz., R. 2003a, GCNC, 1886

Greiner, J., Ries, C, Barwig, H., Fynbo, J., \& Klose, S. 2003b, GCNC, 1894 
Guarnieri, A., Cortese, L., Bartolini, C., et al. 2003, GCNC, 1892

Guilloteau, S., Delannoy, J., Downes, D., et al. 1992, A\&A, 262, 624

Harrison, F. A., Bloom, J. S., Frail, D. A., et al. 1999, ApJ, 523, L121

Harrison, F. A., Yost, S. A., Sari, R., et al. 2001, 559, 123

Henden, A. 2003, GCNC, 1916

Jakobsson, P., Hjorth, J., Fynbo, J. U., et al. 2003, A\&A, 408, 941

Jaunsen, A. O., Hjorth., J., Björnsson, G., et al. 2001, ApJ, 546, 127

Klose, S., Stecklum, B., Zeh, A., et al. 2003, GCNC, 1923

Kulkarni, S. R., Fox, D. W., Berger, E., \& Soderberg, A. M., et al. 2003, GCNC, 1911

Landolt, A. R. 1992, AJ, 104, 340

Maiorano, E., Masetti, N., Palazzi, E., et al. 2003, GCNC, 1933

Masetti, N., Palazzi, E., Pian, E., et al. 2001, A\&A, 374, 382

Mathis, J. S. 1990, ARA\&A, 28, 37

Mészáros P. 2002, ARA\&A, 40, 137

Nysewander, M. C., Moran, J., Reichart, D., \& Schwartz, M. 2003, GCNC, 1921

Pandey, S. B., Sagar, R., Mohan, V., et al. 2001, BASI, 29, 459

Pedersen, K., Fynbo, J., Hjorth, J., \& Watson, D., et al. 2003, GCNC, 1924

Price, P. A., \& Warren, B. E. 2003, GCNC, 1890

Price, P. A., Fox, D. W., \& Chen, H. W. 2003, GCNC, 1880
Price, P. A., Fox, D. W., Djorgovski, S. G., et al. 2003, GCNC, 1889 Rhoads, J. E. 1999, ApJ, 525, 737

Rhoads, J. E., \& Fruchter, A. 2001, ApJ, 546, 117

Rumyantsev, V., Biryukov, V., \& Pozanenko, A. 2003a, GCNC, 1908

Rumyantsev, V., Sergeeva, L., \& Pozanenko, A. 2003b, GCNC, 1929

Sako, M., \& Fox, D. W. 2003, GCNC, 1928

Sagar, R. 2002, BASI, 30, 237

Sagar, R., Mohan, V., Pandey, S. B., et al. 2000, BASI, 28, 499

Sagar, R., Pandey, S. B., Mohan, V., Bhattacharya, D., \& Castro-Tirado, A. J. 2001a, BASI, 29, 1

Sagar, R., Stalin, C. S., Bhattacharya, D., et al. 2001b, BASI, 29, 91

Sari, R., Piran, T., \& Halpern, J. P. 1999, ApJ, 519, L17

Schlegel, D. J., Finkbeiner, D. P., \& Davis, M. 1998, ApJ, 500, 525

Schwarz, P. R. 1980, PASP, 535, 534

Semkov, E. 2003, GCNC, 1935

Stanek, K. Z., Garnavich, P. M., Kaluzny, J., Pych, W., \& Thompson, I. 1999, ApJ, 522, L39

Stanek, K. Z., Krzysztof, Z., Garnavich, P. M., et al. 2001, ApJ, 563, 592

Suzuki, M., Shirasaki, Y., Graziani, C., et al. 2003, GCNC, 1888

Von Braun, K., Garnavich, P., \& Stanek, K. 2003, GCNC, 1881 